\title{
An Innovative Procedure for the Rolling Noise Evaluation
}

\author{
Massimo Viscardi ${ }^{1 a}$, Pasquale Napolitano ${ }^{1}$, Stefano Ferraiuolo ${ }^{2}$ \\ ${ }^{1}$ University of Naples "Federico II", Dept. of Industrial Engineering, Via Claudio, 21 - 80125 Napoli - Italy \\ 2 HITACHI RAIL - Via Argine, 425 - 80147, Napoli - ITALY
}

\begin{abstract}
Noise is often generated by pressure changes in the air induced by mechanical vibrations. The study of these phenomena is known as structural acoustics or, in a more fashionable way, virboacoustics. Vibroacoustics is the study of the mechanical waves in structures and how they interact with, and radiate into, adjacent media. In railway the most important noise source, based on fluid and structure interaction is the rolling noise. The aim of the paper is the development and implementation of a numerical method for the rail decay rate and combined roughness calculation according to the FprCEN/TR 16891:2015 and a subsequent evaluation of the excess noise level in accordance with the ISO/FDIS 3095: 2013. The tool, as a final results, will make possible the evaluation of the rail parameters without the involvement of long and expensive test campaign based on classical roughness measurement methods and will permit the compensation of the roughness induced excess noise level for a comparative comprehension of the acoustic experimental data.
\end{abstract}

\section{Rolling Noise Generation}

Between 1970 and 1980 there have been numerous studies on the wheel / rail noise generation, and in this area, the research is still open, especially regarding high speed trains. In this area there are several descriptive terms for various types of wheel/rail noise. The terms rolling noise and tangent track noise are both used here to refer to noise produced by rail and wheel roughness and material heterogeneity. Rolling noise also occurs at curved as well as tangent track. At systems with adequate ground rail and trued wheels, traction motor cooling fan and gearbox noise and undercar aerodynamic noise may contribute significantly to operational noise, and some care must be exercised in identifying the wheel/rail noise component. A representative example of 1/3-octave band wayside noise produced by a Bay Area Rapid Transit (BART) vehicle at various speeds are presented in figure 1 . In this example, traction power equipment produces the peak in the spectrum at $250 \mathrm{~Hz}$. At higher frequencies, wheel/rail noise dominates the spectrum, peaking at about 1,600 Hz. With trued wheels and smooth ground rail on ballast and ties, BART is one of the quietest vehicles in operation at U.S. transit systems. With normal rolling noise, the rail running surface will be free of spalls, checks, pitting, burns, corrugation, or other surface defects, which may not be entirely visible. The wheel and rail provide running surfaces which, under ideal conditions, should have similar characteristics for smoothness and low noise as any anti-friction bearing. From a practical perspective, ideal bearing surfaces are difficult to realize and maintain in track due to lack of lubricant, corrosion and contamination, and dynamic wheel/rail interaction forces.

\section{Rolling Noise Sources}

There are four mechanisms that are suggested in the literature, as the main causes of rolling noise.

These are:

- Rail and wheel roughness,

- Parameter variation, or moduli heterogeneity,

- Creep, and

- Aerodynamic noise.

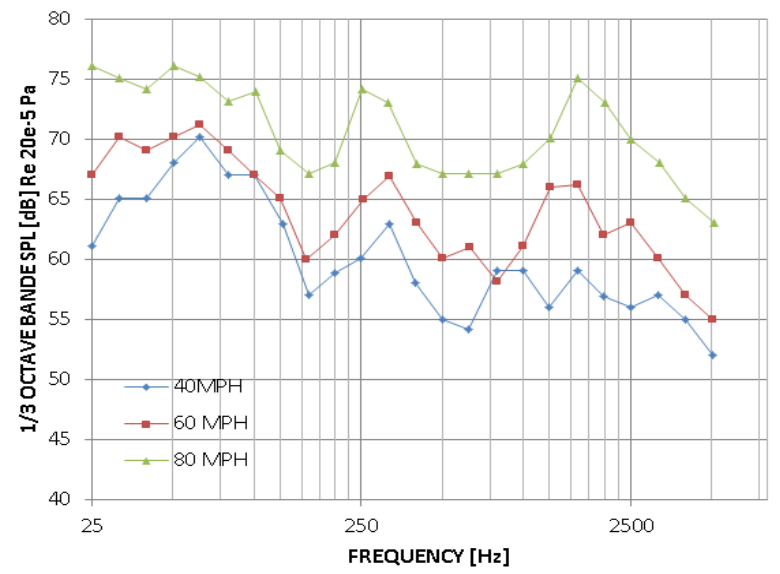

Figure 1: Pass-by noise for single car vehicle with aluminum centered wheels on ballast and tie track.

\footnotetext{
a Corresponding author: massimo.viscardi@unina.it
} 


\subsection{Wheel/Rail Roughness}

Is probably the most significant cause of the wheel/rail noise. The surface roughness profile may be decomposed into a continuous spectrum of wavelengths. At wavelengths short relative to the contact patch dimension, the surface roughness is attenuated by averaging of the roughness across the contact patch, an effect which is described as contact patch filtering. Thus, fine regular grinding marks of dimensions less than, perhaps, $1.5 \mathrm{~mm}$ should not produce significant noise compared to lower frequency components.

\subsection{Parameter Variation}

Parameter variation refers to the variation of rail and wheel steel moduli, rail support stiffness, and contact stiffness due to variation in rail head transverse radius-ofcurvature. The influence of fractional changes in elastic moduli and of radius-of-curvature of the rail head as a function of wavelength necessary to generate wheel/rail noise equivalent to that generated by surface roughness is illustrated in the figure. Experimental data for the effect of modulus variation at this frequency have not yet been found. Rail head ball radius heterogeneity also induces a dynamic response in the wheel and rail. The variation of rail head curvature would have to be -on the order of $10 \%$ to $50 \%$ to produce a noise level similar to that produced by rail roughness alone. Data on rail head radii of curvature as a function of wavelength have not been obtained nor correlated with wayside noise. Also, railhead ball radius variation will normally accompany surface roughness, so that distinguishing between ball radius variation and roughness may be difficult in practice.

\subsection{Dynamic Creep.}

Dynamic creep may include both longitudinal and lateral dynamic creep, roll-slip in a direction parallel with the rail, and spin-creep of the wheel about a vertical axis normal to the wheel/rail contact area.

\subsubsection{Longitudinal Creep}

It is not considered significant by some researchers, as rolling noise levels are claimed to not increase significantly during braking or acceleration on smooth ground rail. However, qualitative changes of the sound of wheel/rail noise on newly ground rail with a grinding pattern in the rail running surface is observable to the ear as a train accelerates or decelerates, in contradiction to the notion that longitudinal creep is of no significance.

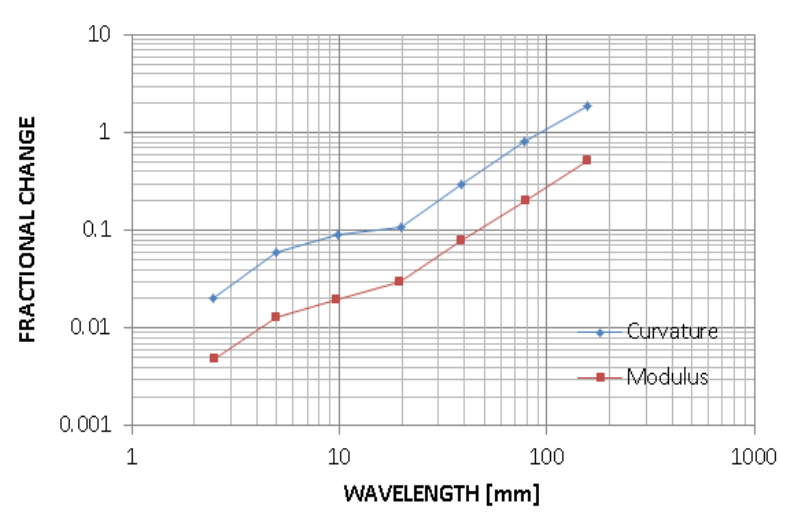

Figure 2: Change in elastic modules and railhead curvature required to generate wheel/rail excitation.

\subsubsection{Lateral creep}

It occurs during curve negotiation, and is responsible for the well-known wheel squeal phenomena resulting from stick-slip. Lateral creep may not be significant at tangent track, but lateral dynamic creep may occur during unloading cycles at high frequencies on abnormally rough or corrugated rail. Lateral dynamic creep is postulated by some to be responsible for short-pitch corrugation at tangent track. Therefore, lateral creep, at least in the broad sense, may be a significant source of noise.

\subsubsection{Spin-creep}

It is caused by wheel taper which produces a rolling radius differential between the field and gauge sides of the contact patch.

\subsection{Aerodynamic Noise}

Aerodynamic noise is caused by turbulent boundary layer noise about the wheel circumference as it moves forward and by undercar components which exhibit substantial aerodynamic roughness. Noise due to air turbulence about the wheel is usually not significant at train speeds representative of transit systems, while noise due to air turbulence in the truck area may be significant.

\section{Tool Introduction}

The tool has been developed according to the FprCEN/TR 16891:2015 in which the whole procedure is specified. In the procedure is described both the analytical procedure for decay rate and roughness calculation and the procedure for FRF estimation. Before show the tool implementation we will investigate the preliminary data needed to obtains results according to the regulation. At the end of the tool a second step, not mentioned in above standard, has been implemented for noise correction and prevision. The extra part developed want to answer at the following question: knowing the FRF of system how change the noise as function of roughness? The answer to this question acquire relevance for both train manufacturer and train regulation institution. In fact, the 
rolling noise maximum level, is given referred to rail with standard roughness curve reported in ISO/FDIS 3095.

\subsection{Input Parameter}

To detect the parameters, we need instruments that allow us, during the passage of the train, to detect not only the useful data to insert in the calculation formulas, but also the standard parameters of the train, as the number of axles and the speed of train during the pass-by. This instrumentation, is constituted by an accelerometer that can be mounted below the longitudinal axis, at the base of the rail, if you need only measure the vertical decay rate, otherwise, another accelerometer is positioned on the side of the railhead as shown in figure a, if you wanted to calculate the lateral decay rate. The number of accelerometers can also be increased if you need more measurements. The position $b$ is more difficult. Indeed in that case we will have an angle which should be filled with the use of an angled pin so to ensure the verticality of the accelerometer. At the end of procedure, positioning the accelerometer as already illustrated, and using the methods described into ISO 5348 to mount the instrumentation, the result is shown in Figure 4. During the pass-by of the train, the instrumentation will detect:

- Vertical railhead vibration (acceleration signal) including the approach and departure of the train;

- $\quad$ Sound pressure time signal, if a transfer function is required;

- $\quad$ Train speed v;

- $\quad$ Train length $\ell$, usually determined from known vehicle lengths;

- $\quad$ Number of axles $\mathrm{N}_{\mathrm{ax}}$, counted or estimated from the vibration or trigger signal;

- $\quad$ Optionally the axle pass-by trigger signal $\mathrm{z}(\mathrm{t})$.

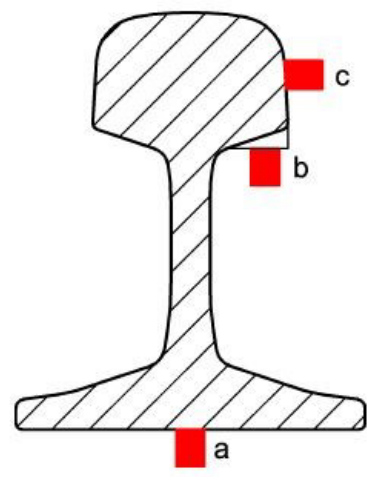

Figure 3: Suitable position of accelerometers

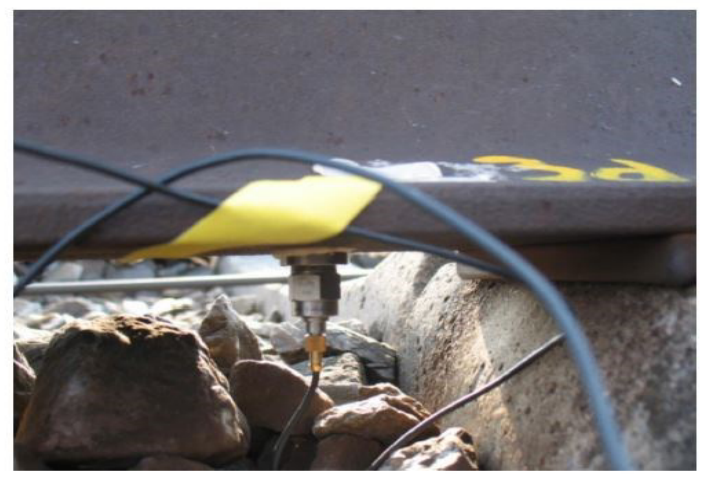

Figure 4: An accelerometer correctly positioned

\section{Decay Rate Evaluation}

To determine the track decay rate, an iterative method very close to that described in the standard EN 15461 is used. The rail vibration amplitude due to a single wheel is assumed to be described by an exponential function

$\mathrm{A}(\mathrm{x}) \approx \mathrm{A}(0) \mathrm{e}^{\beta \mathrm{x}}$

Where $\mathrm{x}$ is the position away from the contact point along the rail; $\mathrm{A}(\mathrm{x})$ is the vibration amplitude along the rail; $A(0)$ is the instantaneous amplitude at the position of the wheel contact point; $\beta$ is a decay exponent. The decay rate in $\mathrm{dB} / \mathrm{m}$ can be given as:

$\mathrm{D}_{\mathrm{z}}=20 \log _{10}\left(\mathrm{e}^{\beta}\right) \approx 8,686 \beta$

This decay rate is derived from the evaluation of the ratio of the integrated vibration energy over a length L2, potentially including the whole train pass-by versus the integrated vibration energy over a short length L1 directly around the wheels. L1 is taken as the shortest axle distance in the train (or part of the train).

A common minimum wheel distance is $1.8 \mathrm{~m}$, in which case the analysis length $\mathrm{L} 1$ extends from $-0,9 \mathrm{~m}$ to $+0,9 \mathrm{~m}$ around each wheel position. In summary, we have two quantities $\mathrm{A}_{\Sigma \mathrm{L} 1}{ }^{2}$ and $\mathrm{A}_{\Sigma \mathrm{L} 2}{ }^{2}$ that can be derived easily from the measurements of the acceleration signals.

We are interested to know how much is their ratio, which gives us the vibration energy ratio $\mathrm{R}$ for each third octave band frequency $f_{c}$.

$\mathrm{R}\left(\mathrm{f}_{\mathrm{c}}\right)=\frac{\mathrm{A}_{\Sigma \mathrm{L} 1}^{2}}{\mathrm{~A}_{\Sigma \mathrm{L} 2}^{2}} \approx 1-\mathrm{e}^{-\beta \mathrm{L} 1}$

We can see, in Figure 5, a typical pass-by spectrum measured by an accelerometer on the head of the rail along vertical direction. In Figure 6 a zoom of signal around a wheel for time $T_{x}$. 


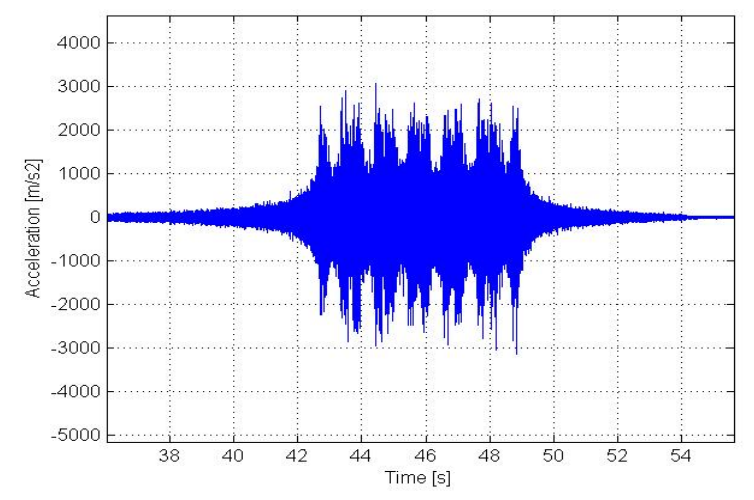

Figure 5: Time signal of vertical rail vibration; unfiltered time signal of whole pass-by with total integration time $\mathrm{T}$ indicated.

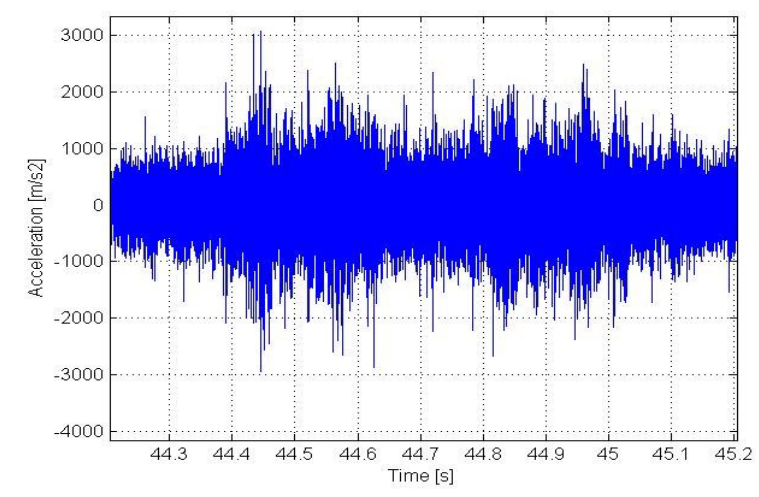

Figure 6: Time signal of vertical rail vibration; selected part of time signal indicated showing integration time $T_{x}$ around each wheel.

\subsection{Iteration Method}

The $T_{x}$ interval contains mainly energy from the single wheel, but also contributions from other wheels, particularly the nearby ones. $\mathrm{T}_{\mathrm{x}}$ should be chosen slightly less than the smallest distance between wheels over the whole train to avoid overlap in energy summation. We can now derive very useful formulas for the purpose of the iterative process, whose steps are shown on the FprCEN/TR 16891:2015 standard. These formulas are:

$\beta_{k}\left(f_{c}\right)=-\frac{\ln \left(1-\frac{R\left(f_{c}\right) N_{a x}}{w_{k}\left(f_{c}\right)}\right)}{L 1}$

$\mathrm{w}_{\mathrm{k}}\left(\mathrm{f}_{\mathrm{c}}\right)=\sum_{\mathrm{j}=1}^{\mathrm{N}_{\mathrm{ax}}} \quad \sum_{\mathrm{i}=1}^{\mathrm{N}_{\mathrm{ax}}} \mathrm{e}^{-2 \beta_{\mathrm{k}-1}\left|\mathrm{x}_{\mathrm{j}}-\mathrm{x}_{\mathrm{i}}\right|}$

The difference $\mathrm{x}_{\mathrm{j}}-\mathrm{x}_{\mathrm{i}}$ is the distance between the current wheel $j$ and another wheel $i$. The weighting coefficient $w_{k}$ represents a sum of the squared contributions from all wheels, viewed from each wheel and then summated over all wheels. If the decay exponent is large, the effect of adjacent wheels is small and $w_{k}$ quickly converges to

$\mathrm{w}_{\mathrm{k}}=\mathrm{N}_{\mathrm{ax}}$
If the decay is small then $\mathrm{w}_{\mathrm{k}}$ becomes larger. Sufficient convergence is often achieved within around five steps.

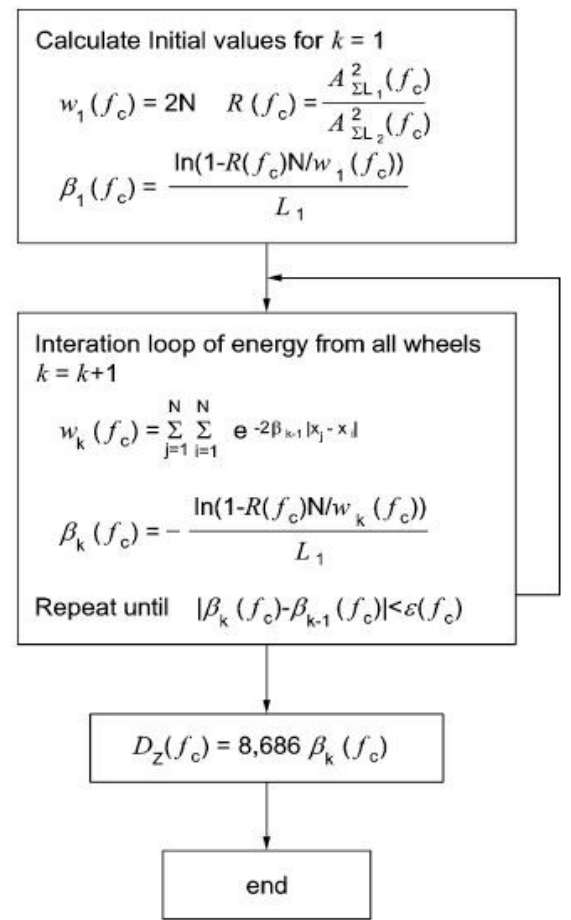

Figure 7: Logic of the energy iteration loop

The cycle showed in figure 7 is the core of standard and provide an accurate way for decay rate estimation. Usually the error $\varepsilon\left(f_{c}\right)$ is 0.1 ; a smaller value can be settled for an higher precision. At the end of the loop we have the decay rate as function of frequency. A typical curve of decay rate in $\mathrm{dB} / \mathrm{m}$ is shown in figure 8 .

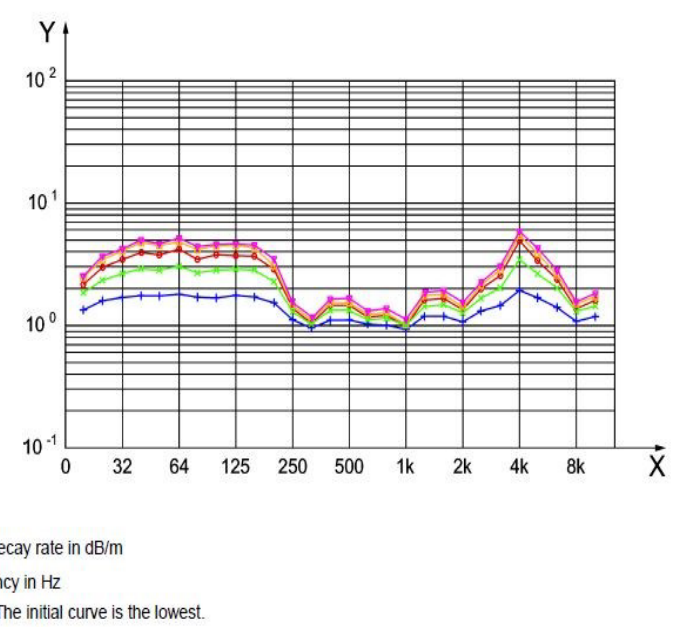

Figure 8: Convergence of a decay rate applying the iteration procedure

\section{Combined Roughness Evaluation}

The combined roughness for the whole train or part of a train with length $\ell$ is determined by the following formula: 
$\mathrm{L}_{\mathrm{Rtot}\left(\mathrm{f}_{\mathrm{c}}\right)}=\mathrm{L}_{\mathrm{aeq}, \mathrm{tp}}\left(\mathrm{f}_{\mathrm{c}}, \mathrm{v}\right)+10 \lg \left(\frac{\mathrm{D}_{\mathrm{z}}\left(\mathrm{f}_{\mathrm{c}}\right) \ell}{8,686 \mathrm{~N}_{\mathrm{ax}}}\right)-\mathrm{A}_{1}\left(\mathrm{f}_{\mathrm{c}}\right)-$

$A_{2}\left(f_{c}\right)-40 \lg \left(2 \pi f_{c}\right)$

The uncertainty in combined roughness can be reduced by using the average decay rate as input for the calculation of the above formula. If the combined roughness is averaged over several pass-by the averaging is done arithmetically.

\subsection{Wavelength domain}

Usually the roughness curve is reported as function of wavelength and not of frequency. The passage from one domain to another is done according the following equation

$\lambda=\frac{\mathrm{v}}{\mathrm{f}}$

Where $\mathrm{v}$ is the train speed in $\mathrm{m} / \mathrm{s}$ and $\mathrm{f}$ is the frequency, usually a one-third octave band center. According to equation 8 with a single speed is impossible to investigate all the wavelength domain. To cover it completely a passby measure at different speeds must be performed. A general idea of wavelength range a single speed can cover a diagram is shown in figure 9 .

The conversion performed applying equation 8 generates a non-centered spectrum. To normalize the wavelength domain to a standard spectrum a weight procedure must be applied to redefine the energy envelop over a standard one-third octave band spectrum.

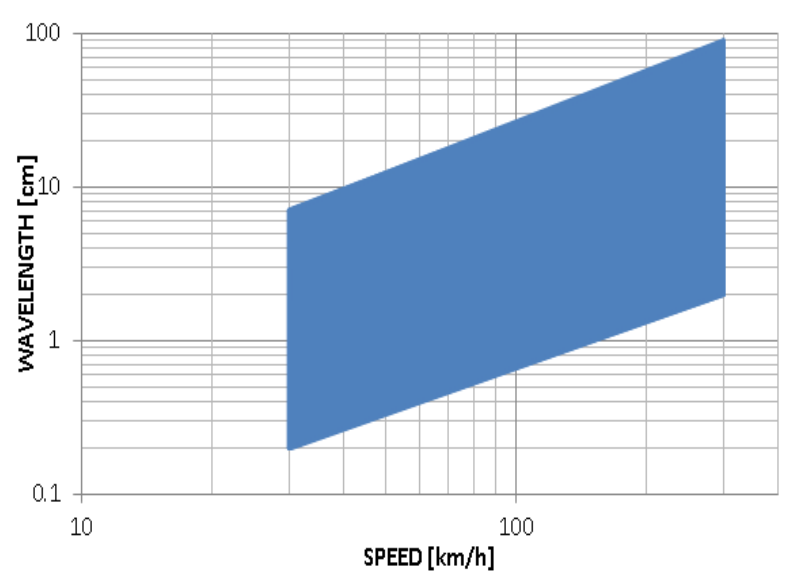

Figure 9: Approximate applicability range for determining combined roughness from rail vibration data during train passby

In table 1 is shown the standard spectrum for wavelength both for third and one-third octave bands.

Table 1: Standard octave and one third octave wavelengths

\begin{tabular}{|c|c|c|c|}
\hline $\begin{array}{c}\text { Octave band centre } \\
\text { wavelength } \\
\text { [mm] } \\
\lambda^{2} \text { c,oct }\end{array}$ & \multicolumn{3}{|c|}{$\begin{array}{c}\text { Third octave band centre } \\
\text { wavelength } \\
\text { [mm] }\end{array}$} \\
\hline 500 & $\lambda^{\prime}$ c,third,1 & $\lambda^{\prime}$ c,third,2 & $\lambda^{\prime}$ c,third,3 \\
\hline 250 & 630 & 500 & 400 \\
\hline 125 & 315 & 250 & 200 \\
\hline 63 & 160 & 125 & 100 \\
\hline 31,50 & 80 & 63 & 50 \\
\hline 16 & 40 & 31,50 & 25 \\
\hline 8 & 20 & 16 & 12,50 \\
\hline 4 & 10 & 8 & 6,30 \\
\hline 2 & 5 & 4 & 3,15 \\
\hline 1 & 2,50 & 2 & 1,60 \\
\hline & 1,25 & 1 & 0,80 \\
\hline
\end{tabular}

To obtain roughness values at the preferred standard wavelengths $\lambda_{c}$ the roughness levels derived at the neighboring wavelengths around a certain desired standard wavelength are used. First the neighboring wavelengths resulting from the frequency-to-wavelength transformation are located, which are named $\lambda_{-}$and $\lambda_{+}$ such that $\lambda_{-}<\lambda_{c}<\lambda_{+}$. Now the roughness level at wavelength $\lambda_{c}$ can be calculated with:

$\mathrm{L}_{\mathrm{Rtot}}\left(\lambda_{\mathrm{c}}\right)=10 \lg \left\{\frac{\lambda_{+}-\lambda_{\mathrm{c}}}{\lambda_{+}-\lambda_{-}} 10^{\frac{\mathrm{L}_{\mathrm{Rtot}}\left(\lambda_{-}\right)}{10}}+\frac{\lambda_{\mathrm{c}}-\lambda_{-}}{\lambda_{+}-\lambda_{-}} 10^{\frac{\mathrm{L}_{\mathrm{Rtot}}\left(\lambda_{+}\right)}{10}}\right\}$

A correct conversion between one domain to another is only possible using the exact center wavelengths $\lambda_{c}$ and center frequencies $\mathrm{f}_{\mathrm{c}}$, as defined in EN ISO 266.

Using the nominal center wavelengths in table above, for the conversion leads to an overlapping of wavelength bands and a small error regarding the conservation of energy in the order of $1 \%$ or less. It may be required to have measurements at more than one speed so as to obtain a sufficient wavelength range for a particular application. Roughness spectra from multiple measurements may be averaged over common wavelengths, omitting or including points outside the common wavelength range.

\section{Acoustic FRF Estimation}

If rolling noise is the only significant source during a train pass-by, we can calculate the transfer function in two ways. The first is determined from the equivalent sound pressure level and combined roughness at speed $\mathrm{v}$, and normalized to the axle density $\mathrm{N}_{\mathrm{ax}} / \mathrm{l}$.

We use the following formula:

$\mathrm{L}_{\text {HpRtot,nl }}\left(\mathrm{f}_{\mathrm{c}}\right)=\mathrm{L}_{\text {peq,tp }}\left(\mathrm{f}_{\mathrm{c}}\right)-\mathrm{L}_{\mathrm{Rtot}}\left(\mathrm{f}_{\mathrm{c}}, \mathrm{v}\right)-10 \lg \left(\frac{\mathrm{N}_{\mathrm{ax}}}{\ell}\right)$ 
The total transfer function L_(HpRtot,nl) (f_c ) is independent from the roughness, train length and number of axles. It characterizes the vibro-acoustic properties of the vehicle, the track and the propagation area.

\subsection{Power FRF Estimation}

The second way is that the transfer function can also be defined in terms of sound power which is given for a defined length of track or vehicle and is normalized to the number of axles:

$\mathrm{L}_{\text {HWRtot, },}\left(\mathrm{f}_{\mathrm{c}}\right)=\mathrm{L}_{\mathrm{W}}\left(\mathrm{f}_{\mathrm{c}}\right)-\mathrm{L}_{\text {Rtot }}\left(\mathrm{f}_{\mathrm{c}}, \mathrm{v}\right)-10 \lg \left(\mathrm{N}_{\mathrm{ax}}\right)$

\section{Correction of the measured noise}

ISO 3095 provides a reference roughness curve that represents the value should not be exceeded. Otherwise the standard admits a higher value and in fact the same ISO 3095 present a corrected roughness obtained with the following formula

$\mathrm{L}_{\mathrm{c}}\left(\mathrm{f}_{\mathrm{c}}\right)=\min \left[\mathrm{L}_{\mathrm{m}}\left(\mathrm{f}_{\mathrm{c}}\right), \mathrm{L}_{\mathrm{ISO}}\left(\mathrm{f}_{\mathrm{c}}\right)\right]$

Where $\mathrm{L}_{\mathrm{m}}$ indicate the measured roughness and $\mathrm{L}_{\mathrm{ISO}}$ the standard one. In standard the equation 12 in expressed in wavelength domain but it cans be converted to frequency one.

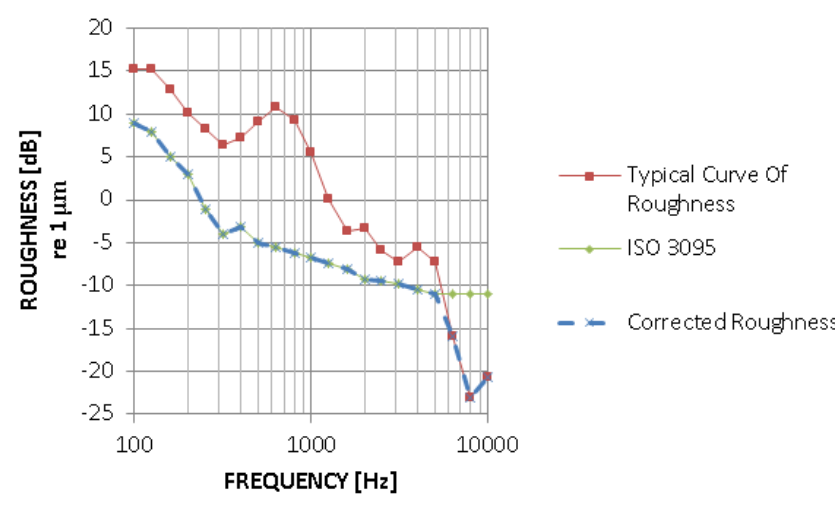

Figure 10: Typical roughness curve and standard one comparison; corrected roughness calculated according ISO 3095;

\section{Conclusions}

The tool described above and its implementation seems to offer a robust and affordable method for roughness evaluation. The tool provides a fast way to obtain a complex parameter starting from accelerometers measurements. The tool has two limits; first it results in a wheel/rail combined roughness and it doesn't provide any way to divide the rail roughness from wheel one. Second, the FRF calculated is independent from the train, wheel and rail types. It is a characteristic of the system and can't be used to provide data for other trains on the same rail or, that is the same, for that train on different rails. According to these limitations the tool remains a good way for roughness estimation for its simplicity and strength. Another approach of this standard is, surely, for rail realtime monitoring. A fixed accelerometer can analyzes trains pass-by for long period and advice when the roughness exceeds the threshold value for safety and noise comfort.

\section{References}

[1] Ente Italiano di Normazione, UNI/CT 050 , FprCEN/TR 16891 "Railway applications Acoustics - Measurement method for combined roughness, track decay rates and transfer functions".

[2] ISO/FDIS 3095: 2013 "Acoustics - Railway applications - Measurement of noise emitted by railbound vehicles"

[3] Thompson, D.J., Wheel-Rail Noise Generation, Part I: Introduction and Interaction Model, Journal of Sound and Vibration,161, 1993a.

[4] Thompson, D.J., Wheel-Rail Noise Generation, Part II: Wheel Vibration, Journal of Sound and Vibration, 161, 1993b.

[5] Thompson, D.J., Wheel-Rail Noise Generation, Part I: Inclusion of wheel rotation, Journal of Sound and Vibration, 161, 1993c

[6] Thompson, D.J., Experimental Analysis of Wave Propagation in Railway Propagation in Railway Track, Journal of Sound and Vibration, 203(5), 1997.

[7] James T. Nelson, Transit Cooperative Research Program - Wheel/Rail Noise Control Manual TCRP report 23.

[8] Rusciano, N., Viscardi, M., Ferraiuolo, S. Vibro acoustic characterization of a light metro rail traction system (2006) 13th International Congress on Sound and Vibration 2006, ICSV 2006, 7, pp. 5366-5374.

[9] Rusciano N., Scarselli G., Viscardi M., Ferraiolo S., Lecce L. "Vibro-acoustic characterization of a light metro vehicle (2003) Acta Acustica (Stuttgart), 89 (SUPP.), pp. S76.

[10] Iadevaia M., Viscardi M., Ferraiuolo S. "Structural vibration and sound power radiation modelling for a light train wheel" (2003) Proceedings of the Tenth International Congress on Sound and Vibration, pp. 1743-1748. 